\title{
Comparative efficacy of autologous versus cadaveric saphenous vein grafts in cerebral revascularization surgery
}

\author{
Christopher J. Stapleton, MD, Ahmed E. Hussein, MD, Mandana Behbahani, MD, Ali Alaraj, MD, \\ Sepideh Amin-Hanjani, MD, and Fady T. Charbel, MD
}

Department of Neurosurgery, University of Illinois at Chicago, Illinois

OBJECTIVE Cerebral bypasses are performed for the purpose of either flow augmentation for ischemic cerebrovascular disease or flow replacement for vessel sacrifice during complex aneurysm or tumor surgery. Saphenous vein grafts (SVGs) are commonly used interposition grafts. The authors of this study sought to compare the efficacy of autologous versus cadaveric SVGs in a large series of cerebral bypasses using interposition vein grafts with long-term angiographic follow-up.

METHODS All intracranial bypass procedures performed between 2001 and 2018 were reviewed. Demographic, clinical, angiographic, and operative data were recorded and then analyzed according to SVG type.

RESULTS A total of 308 consecutive intracranial bypasses were performed during the study period, $53(17.2 \%)$ of which were bypasses with an interposition SVG (38 autologous, 15 cadaveric). At a median follow-up of 2.2 months (IQR 0.229.1), 39 (73.6\%) bypasses were patent (26 [68.4\%] autologous, 13 [86.7\%] cadaveric, $p=0.30)$. Comparing autologous and cadaveric SVG recipients, there were no statistically significant differences in age $(p=0.50)$, sex $(p>0.99)$, history of smoking $(p=0.75)$, hypertension $(p>0.99)$, diabetes mellitus $(p=0.13)$, indication for bypass $(p=0.27)$, or SVG diameter $(p=0.65$ ). While there were higher intraoperative (autologous, $100.0 \mathrm{ml} / \mathrm{min}, \mathrm{IQR} 84.3-147.5$; cadaveric, 80.0 $\mathrm{ml} / \mathrm{min}, \mathrm{IQR}$ 47.3-107.8; $\mathrm{p}=0.11$ ) and postoperative (autologous, $142.2 \mathrm{ml} / \mathrm{min}, \mathrm{IQR}$ 76.8-160.8; cadaveric, $92.0 \mathrm{ml} / \mathrm{min}$, IQR 69.2-132.2; $p=0.42$ ) volumetric flow rates in the autologous SVGs compared to those in the cadaveric SVGs, the difference between the two groups did not reach statistical significance. In addition, the blood flow index, or ratio of postoperative to intraoperative blood flow, for each bypass was similar between the groups (autologous, 1.3, IQR 0.9-1.6; cadaveric, 1.5, IQR 1.0-2.3; $p=0.37$ ). Kaplan-Meier estimates showed no difference in bypass patency rates over time between autologous and cadaveric SVGs $(p=0.58)$.

CONCLUSIONS Cadaveric SVGs are a reasonable interposition graft option in cerebral revascularization surgery when autologous grafts are not available.

https://thejns.org/doi/abs/10.3171/2020.3.JNS192546

KEYWORDS cerebral blood flow; cerebral ischemia; extracranial-intracranial bypass; intracranial aneurysm; saphenous vein graft; vascular disorders

$\mathrm{C}$ EREBRAL revascularization remains an important aspect of neurovascular surgery. ${ }^{1}$ In flow augmentation and flow replacement surgery, the use of interposition grafts is sometimes necessary when native donor vessels, such as the superficial temporal artery, are inadequate for either flow or length purposes. ${ }^{2,3}$ The saphenous vein and radial artery are commonly used autologous interposition grafts. ${ }^{4-6}$ Cadaveric, cryopreserved saphenous vein grafts (SVGs) have been used extensively in peripheral vascular ${ }^{7-13}$ and cardiac surgery. ${ }^{14-18}$ We previously reported our preliminary experience using cadaveric SVGs in 10 cerebral revascularization cases and noted a short-term patency rate of $100 \% .{ }^{19}$ However, long-term patency rates and efficacy compared to those for autologous SVGs remain unknown. In the present report, we sought to compare demographic, clinical, angiographic, and hemodynamic data from cerebral bypasses with autologous or cadaveric SVGs. 


\section{Methods}

\section{Clinical Cohort}

After receiving approval from the University of Illinois at Chicago Institutional Review Board, we performed a retrospective review of all intracranial bypass operations performed at the University of Illinois at Chicago between January 2001 and December 2018. Demographic, clinical, angiographic, and operative data were collected from the electronic medical record.

Patients with atherosclerotic disease were considered suitable candidates for revascularization if the following criteria were met: 1) symptomatic steno-occlusive disease affecting the anterior or posterior circulation with stroke or transient ischemic attack despite maximal medical therapy; 2) lack of medical comorbidities precluding surgery; and 3) evidence of impaired cerebrovascular hemodynamics.

\section{Materials}

$\mathrm{ABO} / \mathrm{Rh}$ blood group-compatible cadaveric, cryopreserved SVGs were obtained from CryoLife Inc. Veins were harvested from single cadavers using a "no-touch" technique, kept moist with Dulbecco's modified Eagle medium ( $10 \%$ bovine serum, $0.12 \mathrm{mg} / \mathrm{ml}$ papaverine), then cryopreserved and stored at $-196^{\circ} \mathrm{C}$ in a solution of dimethyl sulfoxide. ${ }^{20}$ At surgery, the graft was then thawed and diluted over 30-45 minutes prior to preparation and implantation; all patients were given $1 \mathrm{~g}$ of methylprednisolone intraoperatively.

\section{Blood Flow, Vessel Diameter, and Wall Shear Stress Measurements}

Intraoperative blood flow measurements were made using an ultrasonic flow probe (Charbel Micro-Flow Probe, Transonic Systems Inc.) that provides flow quantification using the principle of ultrasonic transit time to sense flow in vessels independent of the flow velocity profile, turbulence, or hematocrit. ${ }^{21}$ The probe is manufactured in a variety of sizes to accommodate vessel width. Postoperative vessel diameter calculations and blood flow measurements were made using quantitative phase-contrast MRA (QMRA). This technique was implemented using the commercially available noninvasive optimal vessel analysis software (NOVA, VasSol Inc.) and has been described and validated previously.22,23 Wall shear stress (WSS; dynes/ $\mathrm{cm}^{2}$ ) was calculated using the Hagen-Poiseuille equation (WSS $=32 \mu Q / \pi D^{3}$ ), where $\mu$ is blood viscosity (assumed to be constant at 0.035 poise), $Q$ is volumetric flow rate $(\mathrm{ml} / \mathrm{min})$, and $D$ is vessel diameter $(\mathrm{cm}) .^{24}$

\section{Statistical Analysis}

Descriptive statistics were calculated for the demographic, clinical, angiographic, and operative factors, using the median (interquartile range) as a measure of central tendency. Comparisons of continuous variables with nonnormal distributions were made using the nonparametric Kruskal-Wallis H-test, and categorical variables were compared using the Fisher exact test or chi-square test. Kaplan-Meier estimates of bypass patency were per- formed using the log-rank (Mantel-Cox) test. Statistical analyses were performed using JMP (version 14, SAS Institute Inc.) and Prism (version 8.0.1, GraphPad Software). Values of $\mathrm{p}<0.05$ were considered significant.

\section{Results}

A total of 308 consecutive intracranial bypasses were performed during the study period, $53(17.2 \%)$ of which were bypasses with an interposition SVG (38 autologous, 15 cadaveric). Preliminary outcome data from 3 (20.0\%) of the 15 cadaveric cases have been previously reported. ${ }^{19}$ Bypass procedures were performed for the purpose of either flow augmentation for steno-occlusive atherosclerotic disease (14 cases) or flow replacement during complex aneurysm (38 cases) or tumor (1 case) surgery. Between autologous and cadaveric SVG recipients, there were no statistically significant differences in age $(p=0.50)$, sex $(\mathrm{p}>0.99)$, history of smoking $(\mathrm{p}=0.75)$, hypertension $(\mathrm{p}>0.99)$, diabetes mellitus $(\mathrm{p}=0.13)$, or indication for bypass $(\mathrm{p}=0.27$; Table 1$)$. There were no reported complications associated with autologous SVG harvest. There were higher intraoperative blood flow rates in autologous (32 cases, $100.0 \mathrm{ml} / \mathrm{min}$, IQR 84.3-147.5) versus cadaveric (14 cases, $80.0 \mathrm{ml} / \mathrm{min}$, IQR 47.3-107.8) SVGs; however, the difference between the two did not rise to the level of statistical significance $(p=0.11)$. Overall bypass patency was not associated with raw intraoperative or postoperative bypass flow rates.

Twenty-nine $(55.8 \%)$ patients underwent postoperative QMRA with NOVA within 1 week of bypass (18 autologous SVGs, 11 cadaveric SVGs). As with intraoperative flow rates, there were higher postoperative flow rates in autologous (142.2 ml/min, IQR 76.8-160.8) versus cadaveric (92.0 $\mathrm{ml} / \mathrm{min}$, IQR 69.2-132.2) SVGs, but again the difference between the two groups was not significant $(p=0.42)$. The blood flow index, or ratio of postoperative to intraoperative blood flow, for each patient did not differ significantly between the groups (autologous, 1.3, IQR 0.9-1.6; cadaveric, 1.5, IQR 1.0-2.3; $\mathrm{p}=0.37$; Fig. 1). As calculated on postoperative QMRA with NOVA, the median diameter of autologous SVGs was $4.4 \mathrm{~mm}$ (IQR 3.7-4.9) compared with $4.6 \mathrm{~mm}$ (IQR 4.0-5.1) for cadaveric SVGs ( $\mathrm{p}=$ 0.65 ; Fig. 2). The median WSS for autologous SVGs was 8.1 dynes/ $\mathrm{cm}^{2}$ (IQR 5.4-11.7) compared with 6.1 dynes/ $\mathrm{cm}^{2}$ (IQR 5.1-8.9) for cadaveric SVGs ( $\mathrm{p}=0.28$; Fig. 3). The aforementioned data are summarized in Table 1.

At a median follow-up of 2.2 months (IQR 0.2-29.1), 39 (73.6\%) bypasses were patent (26 [68.4\%] autologous, 13 [86.7\%] cadaveric, $\mathrm{p}=0.30$ ). Kaplan-Meier estimates showed no difference in bypass patency rates over time between autologous and cadaveric SVGs ( $p=0.58$; Fig. 4). Two (5.3\%) patients with autologous SVGs required endovascular balloon angioplasty to maintain graft patency.

Over the course of the entire study period, relative to our institution's overall cerebral revascularization volume, bypasses with interposition grafts were used with declining frequency: 2001-2006, 30.1\%; 2007-2012, 15.6\%; and 2013-2018, 5.7\% (Fig. 5).

\section{Discussion}

In the present report, we demonstrated that cadaveric 
TABLE 1. Demographic, clinical, angiographic, and operative characteristics

\begin{tabular}{|c|c|c|c|}
\hline & Autologous SVG & Cadaveric SVG & p Value* \\
\hline No. of cases & 38 & 15 & \\
\hline Median age in yrs (IQR) & $58.0(50.0-66.3)$ & $56.0(48.0-62.0)$ & 0.50 \\
\hline Male sex & 12 & 5 & $>0.99$ \\
\hline Smoking & 13 & 4 & 0.75 \\
\hline Hypertension & 24 & 10 & $>0.99$ \\
\hline Diabetes mellitus & 2 & 3 & 0.13 \\
\hline Indication & & & 0.27 \\
\hline Aneurysm & 28 & 10 & \\
\hline Atherosclerosis & 10 & 4 & \\
\hline Tumor & 0 & 1 & \\
\hline \multicolumn{4}{|l|}{ Median blood flow in $\mathrm{ml} / \mathrm{min}(\mathrm{IQR})$, no. of cases } \\
\hline Intraop & 100.0 (84.3-147.5), 32 & 80.0 (47.3-107.8), 14 & 0.11 \\
\hline 1st postop & $142.2(76.8-160.8), 18$ & 92.0 (69.2-132.2), 11 & 0.42 \\
\hline Last postop† & 94.0 (61.4-139), 17 & $64.0(32.0-90.0), 3$ & 0.17 \\
\hline Median blood flow index (IQR), no. of cases $\ddagger$ & $1.3(0.9-1.6), 15$ & $1.5(1.0-2.3), 10$ & 0.37 \\
\hline Median SVG diameter in mm (IQR), no. of cases & $4.4(3.7-4.9), 18$ & $4.6(4.0-5.1), 11$ & 0.65 \\
\hline Median WSS in dynes $/ \mathrm{cm}^{2}(\mathrm{IQR})$, no. of cases & $8.1(5.4-11.7), 18$ & $6.1(5.1-8.9), 11$ & 0.28 \\
\hline Blood flow $<40 \mathrm{ml} / \mathrm{min} \S$ & 6 & 2 & 0.68 \\
\hline Patent & 26 & 13 & 0.30 \\
\hline
\end{tabular}

SVGs perform similarly to autologous SVGs in cerebral bypasses conducted for either flow augmentation or flow replacement purposes. In our analysis of numerous clinical, angiographic, hemodynamic, and outcome parameters, we identified no significant differences between autologous and cadaveric SVGs. While there were higher intraoperative and postoperative volumetric flow rates in autologous SVGs and higher patency rates in cadaveric SVGs, these differences did not rise to the level of statistical significance.

The central concern with regard to the use of cadaveric interposition grafts for revascularization procedures is the observation of reduced long-term (i.e., beyond 1 year) patency rates compared to those with autologous grafts in cardiac and peripheral vascular procedures. ${ }^{7-18}$ For instance, Sellke et al. compared patency rates following coronary artery bypass surgery with cadaveric SVGs in 10 patients and with autologous internal mammary artery (IMA) grafts in 7 patients. ${ }^{16}$ While 7 (100\%) of the IMA grafts were patent at a follow-up range of 2-18 months after surgery, only $6(60.0 \%)$ of the cadaveric SVGs were patent at 1-8 weeks' follow-up and none were patent at 6-30 months' follow-up, even though all had been patent at the time of surgery. Similarly, following 80 procedures for lower-extremity ischemia in 76 patients, Harris et al. found that $36.8 \%$ of cadaveric SVGs were patent at 1 year, whereas only $23.6 \%$ were patent at 3 years. ${ }^{12}$ Our preliminary report on the use of cadaveric SVGs in cerebral revascularization surgery noted a $100 \%$ short-term graft patency rate. ${ }^{19}$ In the present analysis, at a median follow- up of 2.2 months (IQR 0.2-29.1), 13 (86.7\%) of 15 cadaveric SVGs were patent compared with $26(68.4 \%)$ of 38 autologous SVGs $(\mathrm{p}=0.30)$, and Kaplan-Meier estimates showed no difference in the bypass patency rate over time between SVG types $(\mathrm{p}=0.58)$. In addition, of the 13 cadaveric SVGs patent beyond the 1st postoperative day, 13 $(100 \%)$ were patent at the last follow-up.

Commonly used interposition graft options for cerebral bypasses include SVGs and radial artery grafts, although use of the cephalic vein, ${ }^{25}$ internal maxillary artery, ${ }^{26,27}$

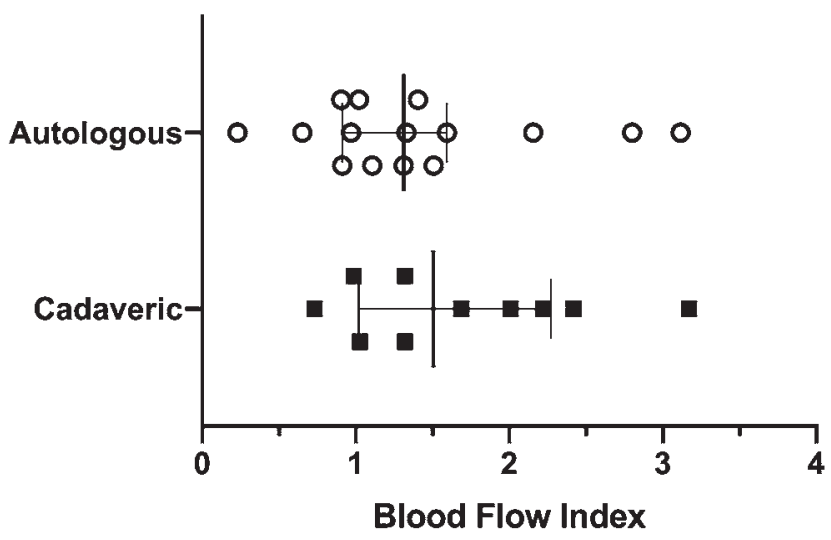

FIG. 1. Individual blood flow index values for 15 autologous (median 1.3, IQR $0.9-1.6$ ) and 10 cadaveric (median 1.5, IQR 1.0-2.3) SVGs $(p=$ $0.37)$. 


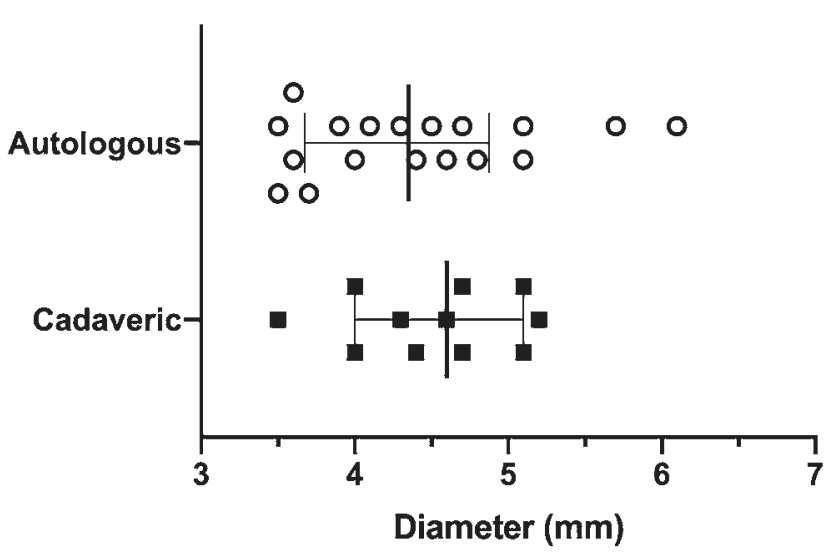

FIG. 2. Individual graft diameter values for 18 autologous (median 4.4 , IQR 3.7-4.9) and 11 cadaveric (median 4.6, IQR 4.0-5.1) SVGs ( $p=$ $0.65)$.

and descending branch of the lateral circumflex femoral artery $^{28}$ has been reported as well. While complications and morbidity associated with saphenous vein ${ }^{29}$ or radial artery $^{30}$ harvest are generally low, these autologous options are sometimes unavailable because of prior harvest or are unsuitable for bypass because of poor quality due to a small size, inherent atherosclerotic disease, or trauma from line insertion. The use of cadaveric SVGs allows selection of a graft of a desired length and diameter and avoids the need for a potentially long extremity incision. The disadvantages of using cadaveric, cryopreserved grafts include lack of immediate availability and concern due to peripheral vascular and cardiac surgical precedents of reduced long-term patency rates, possibly from the use of nonnative tissue and immune-related graft rejection. The mechanism of interposition graft occlusion over time, however, is multifactorial. In their analysis of 159 bypasses using the excimer laser-assisted nonocclusive anastomosis (ELANA) technique, Bremmer et al. noted that bypasses with lower flow (less than $40 \mathrm{ml} / \mathrm{min}$ ) were significantly more likely to occlude over time than bypasses with medium to high flow. ${ }^{31}$ WSS has also been implicated in the pathophysiology of bypass graft occlusion, ${ }^{32}$ such that low WSS is believed to promote myointimal hyperplasia and subsequent graft nonpatency. ${ }^{33}$ With regard to cadaveric SVGs specifically, chronic rejection has also been postulated as a mechanism of occlusion. While $1 \mathrm{~g}$ of methylprednisolone was administered to all patients in this series, the efficacy of steroids or other immunosuppressive agents, such as azathioprine or cyclosporine, with regard to graft patency has not been clearly established..$^{34-37}$

A recent survey of the National Inpatient Sample (NIS) from 2002 to 2014 showed a nearly two-fold increase in the number of cerebral revascularization procedures between the time periods of 2002-2004 and 2011-2014. ${ }^{1}$ However, after the time period from 2008 to 2010, the relative percent of bypass procedures for atherosclerotic disease and aneurysms steadily declined. This trend likely reflects diminished enthusiasm for flow augmentation for atherosclerotic disease following publication of the Carotid Occlusion Surgery Study (COSS) ${ }^{38}$ and the limited necessity for flow replacement in aneurysm or tumor surgery since the introduction of flow diverters to the endovascular market, ${ }^{39}$ two bypass scenarios that frequently required the use of interposition grafts. While analysis of the NIS data did not disclose the use of interposition grafts, ${ }^{1}$ we noted in our series that bypasses with interposition grafts have been used less frequently over time: 2001-2006, 30.1\%; 2007-2012, 15.6\%; and 2013-2018, 5.7\%. In addition, the reduced use of interposition grafts over time in our practice also reflects the adoption of an algorithmic approach to cerebral revascularization surgery. With the "flow-assisted surgical technique," intraoperative blood flow measurements guide bypass decisions, and we have often found that a traditional superficial temporal arterymiddle cerebral artery bypass is sufficient to meet the flow demands of the territory at risk of ischemia., ${ }^{2,3,40-42}$

Beyond cerebral bypasses, cadaveric SVGs can also be

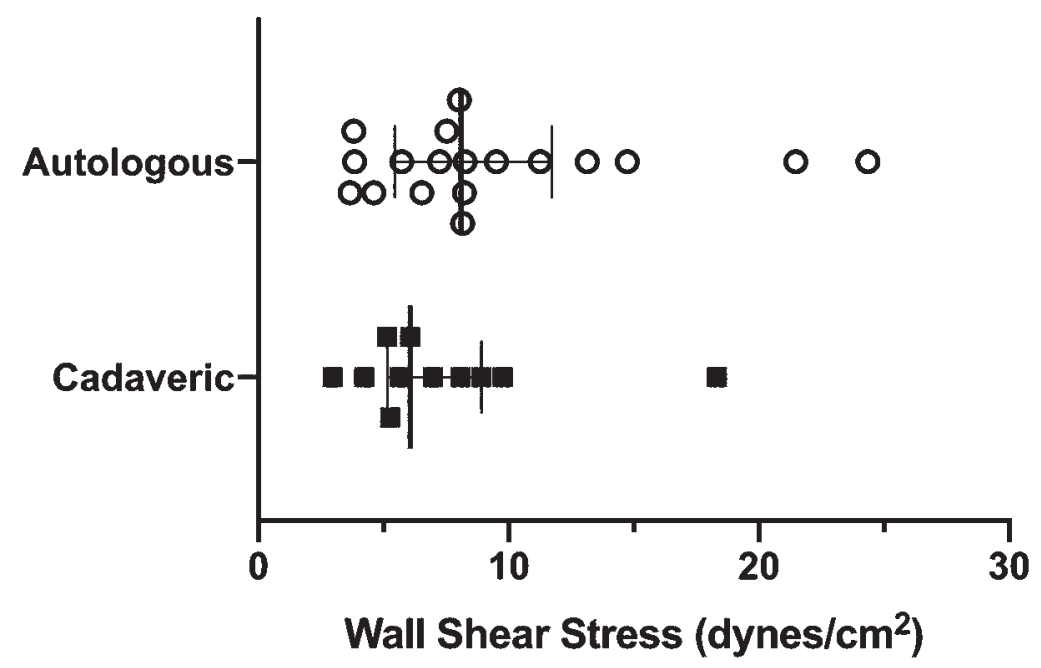

FIG. 3. Individual WSS values for 18 autologous (median 8.1, IQR 5.4-11.7) and 11 cadaveric (median 6.1, IQR 5.1-8.9) SVGs ( $p=$ 0.28). 


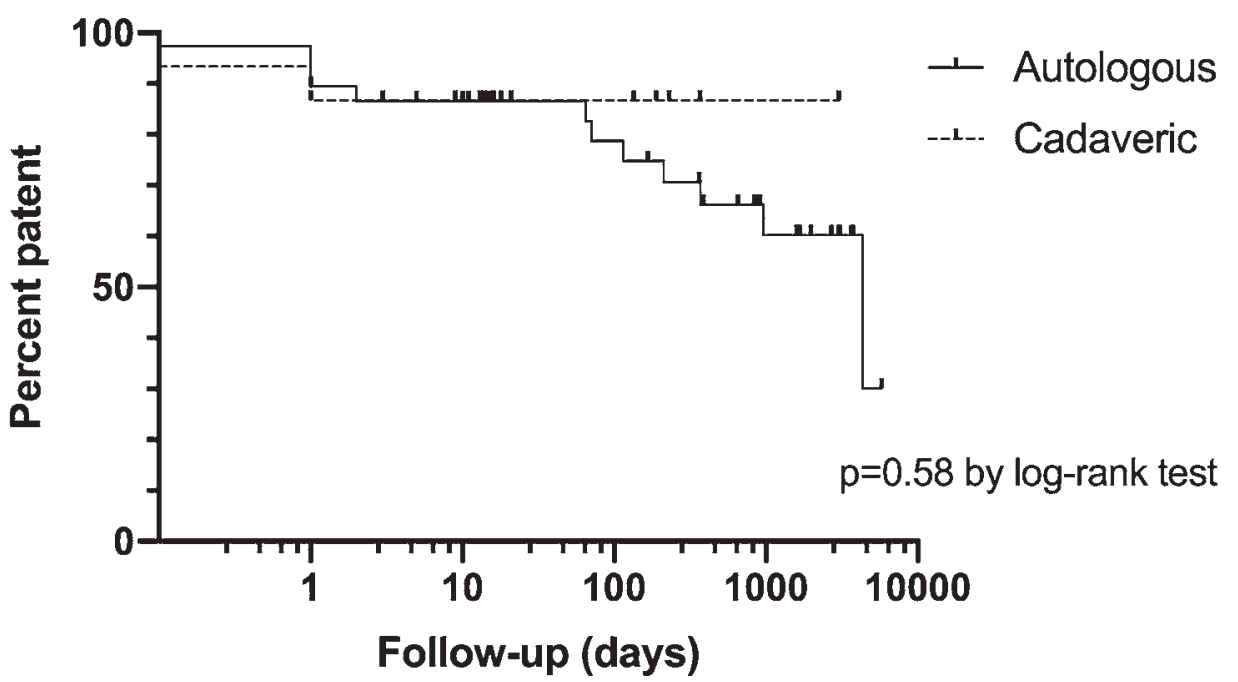

FIG. 4. Kaplan-Meier curves comparing patency over time for bypasses with autologous versus cadaveric SVGs $(p=0.58)$.

useful for the creation of temporary bypasses or conduits. Our experience includes the extracranial construction of a carotid to distal vertebral artery bypass ${ }^{43,44}$ to allow endovascular access via the interposition graft to the distal vertebrobasilar system for aneurysm embolization. ${ }^{45}$

This study has the limitations inherent to any singleinstitution study. While patients were selected for bypass based on institutional protocols, guidelines, and multidis-

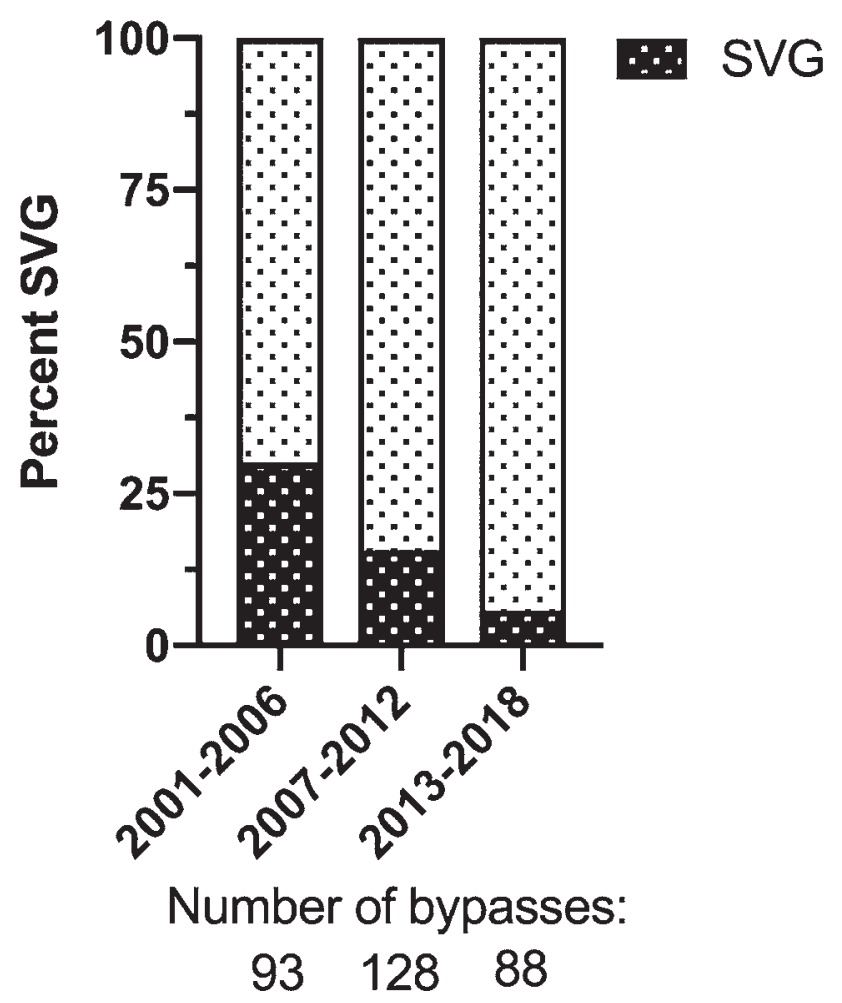

FIG. 5. Utilization of SVGs in cerebral revascularization surgery at one institution from 2001 to 2018. ciplinary consensus, there exists the possibility of selection bias. In addition, the period over which this analysis spans includes recruitment for and publication of the COSS and the US Food and Drug Administration approval of the Pipeline embolization device (Medtronic), which have influenced indications for bypass in atherosclerosis and aneurysm cases, respectively. Despite these considerations, the large number of bypass procedures in the study were performed by two experienced cerebrovascular surgeons (S.A.H. and F.T.C.), and bypass protocols remained similar over the study period.

\section{Conclusions}

In our analysis of numerous clinical, angiographic, hemodynamic, and outcome parameters, we identified no significant differences between autologous and cadaveric SVGs utilized in cerebral bypasses performed for either flow augmentation or flow replacement purposes. As volumetric flow rates and long-term patency rates of autologous and cadaveric SVGs were statistically similar, cadaveric SVGs remain a reasonable interposition graft option when autologous grafts are not available.

\section{References}

1. Winkler EA, Yue JK, Deng H, et al. National trends in cerebral bypass surgery in the United States, 2002-2014. Neurosurg Focus. 2019;46(2):E4.

2. Amin-Hanjani S, Charbel FT. Flow-assisted surgical technique in cerebrovascular surgery. Surg Neurol. 2007;68(suppl 1):S4-S11.

3. Rustemi O, Amin-Hanjani S, Shakur SF, et al. Donor selection in flow replacement bypass surgery for cerebral aneurysms: quantitative analysis of long-term native donor flow sufficiency. Neurosurgery. 2016;78(3):332-342.

4. Liu JK, Kan P, Karwande SV, Couldwell WT. Conduits for cerebrovascular bypass and lessons learned from the cardiovascular experience. Neurosurg Focus. 2003;14(3):e3.

5. Kawashima M, Rhoton AL Jr, Tanriover N, et al. Microsur- 
gical anatomy of cerebral revascularization. Part I: anterior circulation. J Neurosurg. 2005;102(1):116-131.

6. Regli L, Piepgras DG, Hansen KK. Late patency of long saphenous vein bypass grafts to the anterior and posterior cerebral circulation. J Neurosurg. 1995;83(5):806-811.

7. Walker PJ, Mitchell RS, McFadden PM, et al. Early experience with cryopreserved saphenous vein allografts as a conduit for complex limb-salvage procedures. J Vasc Surg. 1993;18(4):561-569.

8. Martin RS III, Edwards WH, Mulherin JL Jr, et al. Cryopreserved saphenous vein allografts for below-knee lower extremity revascularization. Ann Surg. 1994;219(6):664-672.

9. Posner MP, Makhoul RG, Altman M, et al. Early results of infrageniculate arterial reconstruction using cryopreserved homograft saphenous conduit (CADVEIN) and combination low-dose systemic immunosuppression. J Am Coll Surg. 1996;183(3):208-216.

10. Lesèche $\mathrm{G}$, Penna $\mathrm{C}$, Bouttier $\mathrm{S}$, et al. Femorodistal bypass using cryopreserved venous allografts for limb salvage. Ann Vasc Surg. 1997;11(3):230-236.

11. Albertini JN, Barral X, Branchereau A, et al. Long-term results of arterial allograft below-knee bypass grafts for limb salvage: a retrospective multicenter study. J Vasc Surg. 2000;31(3):426-435.

12. Harris L, O'Brien-Irr M, Ricotta JJ. Long-term assessment of cryopreserved vein bypass grafting success. J Vasc Surg. 2001;33(3):528-532.

13. Farber A, Major K, Wagner WH, et al. Cryopreserved saphenous vein allografts in infrainguinal revascularization: analysis of 240 grafts. J Vasc Surg. 2003;38(1):15-21.

14. Ochsner JL, Lawson JD, Eskind SJ, et al. Homologous veins as an arterial substitute: long-term results. J Vasc Surg. 1984;1(2):306-313.

15. Gelbfish J, Jacobowitz IJ, Rose DM, et al. Cryopreserved homologous saphenous vein: early and late patency in coronary artery bypass surgical procedures. Ann Thorac Surg. 1986;42(1):70-73.

16. Sellke FW, Stanford W, Rossi NP. Failure of cryopreserved saphenous vein allografts following coronary artery bypass surgery. J Cardiovasc Surg (Torino). 1991;32(6):820-823.

17. Laub GW, Muralidharan S, Clancy R, et al. Cryopreserved allograft veins as alternative coronary artery bypass conduits: early phase results. Ann Thorac Surg. 1992;54(5):826-831.

18. Iaffaldano RA, Lewis BE, Johnson SA, et al. Patency of cryopreserved saphenous vein grafts as conduits for coronary artery bypass surgery. Chest. 1995;108(3):725-729.

19. Mery FJ, Amin-Hanjani S, Charbel FT. Cerebral revascularization using cadaveric vein grafts. Surg Neurol. 2009;72(4):362-368.

20. Buckley CJ, Abernathy S, Lee SD, et al. Suggested treatment protocol for improving patency of femoral-infrapopliteal cryopreserved saphenous vein allografts. J Vasc Surg. 2000;32(4):731-738.

21. Charbel FT, Hoffman WE, Misra M, Ostergren L. Ultrasonic perivascular flow probe: technique and application in neurosurgery. Neurol Res. 1998;20(5):439-442.

22. Zhao M, Charbel FT, Alperin N, et al. Improved phase-contrast flow quantification by three-dimensional vessel localization. Magn Reson Imaging. 2000;18(6):697-706.

23. Calderon-Arnulphi M, Amin-Hanjani S, Alaraj A, et al. In vivo evaluation of quantitative MR angiography in a canine carotid artery stenosis model. AJNR Am J Neuroradiol. 2011;32(8):1552-1559.

24. Zhao X, Zhao M, Amin-Hanjani S, et al. Wall shear stress in major cerebral arteries as a function of age and gender-a study of 301 healthy volunteers. J Neuroimaging. 2015;25(3):403-407.

25. Nossek E, Costantino PD, Chalif DJ, et al. Forearm cephalic vein graft for short, "middle"-flow, internal maxillary artery to middle cerebral artery bypass. Oper Neurosurg (Hagerstown). 2016;12(2):99-105.

26. Nossek E, Costantino PD, Eisenberg M, et al. Internal maxillary artery-middle cerebral artery bypass: infratemporal approach for subcranial-intracranial (SC-IC) bypass. Neurosurgery. 2014;75(1):87-95.

27. Wang L, Cai L, Lu S, et al. The history and evolution of internal maxillary artery bypass. World Neurosurg. 2018;113:320-332.

28. Başkaya MK, Kiehn MW, Ahmed AS, et al. Alternative vascular graft for extracranial-intracranial bypass surgery: descending branch of the lateral circumflex femoral artery. Neurosurg Focus. 2008;24(2):E8.

29. Zenati MA, Bhatt DL, Stock EM. Endoscopic versus open vein-graft harvesting for CABG. $N$ Engl J Med. 2019;380(22):e43.

30. Rahouma M, Kamel M, Benedetto U, et al. Endoscopic versus open radial artery harvesting: a meta-analysis of randomized controlled and propensity matched studies. J Card Surg. 2017;32(6):334-341.

31. Bremmer JP, Verweij BH, Klijn CJ, et al. Predictors of patency of excimer laser-assisted nonocclusive extracranial-tointracranial bypasses. J Neurosurg. 2009;110(5):887-895.

32. Kleinstreuer C, Hyun S, Buchanan JR Jr, et al. Hemodynamic parameters and early intimal thickening in branching blood vessels. Crit Rev Biomed Eng. 2001;29(1):1-64.

33. Jackson M, Wood NB, Zhao S, et al. Low wall shear stress predicts subsequent development of wall hypertrophy in lower limb bypass grafts. Artery Res. 2009;3(1):32-38.

34. Ricotta JJ, Collins GJ Jr, Rich NM, Reynolds DG. Failure of immunosuppression to prolong venous allograft survival. Arch Surg. 1980;115(1):99-101.

35. Miller VM, Bergman RT, Gloviczki P, Brockbank KG. Cryopreserved venous allografts: effects of immunosuppression and antiplatelet therapy on patency and function. J Vasc Surg. 1993;18(2):216-226.

36. Carpenter JP, Tomaszewski JE. Immunosuppression for human saphenous vein allograft bypass surgery: a prospective randomized trial. J Vasc Surg. 1997;26(1):32-42.

37. Carpenter JP, Tomaszewski JE. Human saphenous vein allograft bypass grafts: immune response. J Vasc Surg. 1998;27(3):492-499.

38. Powers WJ, Clarke WR, Grubb RL Jr, et al. Extracranialintracranial bypass surgery for stroke prevention in hemodynamic cerebral ischemia: the Carotid Occlusion Surgery Study randomized trial. JAMA. 2011;306(18):1983-1992. Published correction in JAMA. 2011;306(24):2672.

39. Kallmes DF, Ding YH, Dai D, et al. A new endoluminal, flow-disrupting device for treatment of saccular aneurysms. Stroke. 2007;38(8):2346-2352.

40. Amin-Hanjani S, Du X, Mlinarevich N, et al. The cut flow index: an intraoperative predictor of the success of extracranial-intracranial bypass for occlusive cerebrovascular disease. Neurosurgery. 2005;56(1)(suppl):75-85.

41. Ashley WW, Amin-Hanjani S, Alaraj A, et al. Flow-assisted surgical cerebral revascularization. Neurosurg Focus. 2008;24(2):E20.

42. Amin-Hanjani S, Alaraj A, Charbel FT. Flow replacement bypass for aneurysms: decision-making using intraoperative blood flow measurements. Acta Neurochir (Wien). 2010;152(6):1021-1032.

43. Carney AL, Anderson EM. Carotid distal vertebral bypass for carotid occlusion: case report and technique. Clin Electroencephalogr. 1978;9:105-109.

44. Charbel FT, Alaraj A, Amin-Hanjani S. Extracranial vertebral artery diseases. In: Winn HR, ed. Youmans Neurological Surgery. 6th ed. Vol 4. Elsevier; 2011:3665-3680.

45. Chwajol M, Munson TA, Alaraj A, et al. Extracranial carotidvertebral bypass for endovascular access to complex poste- 
rior circulation aneurysms: a novel management approach. Neurosurgery. 2012;70(5):1296-1304.

\section{Disclosures}

Dr. Charbel is a consultant for Transonic Inc.

\section{Author Contributions}

Conception and design: Stapleton, Alaraj, Amin-Hanjani, Charbel. Acquisition of data: Stapleton, Hussein, Behbahani. Analysis and interpretation of data: Stapleton, Hussein, Behbahani. Drafting the article: Stapleton. Critically revising the article: Stapleton, Hussein, Behbahani, Amin-Hanjani. Reviewed submitted version of manuscript: all authors. Approved the final version of the manuscript on behalf of all authors: Stapleton. Statistical analysis: Stapleton, Hussein, Behbahani. Administrative/technical/material support: Alaraj, Amin-Hanjani, Charbel. Study supervision: Alaraj, Amin-Hanjani, Charbel.

\section{Correspondence}

Fady T. Charbel: University of Illinois at Chicago, IL. fcharbel@ uic.edu. 